Physics

Physics Research Publications

\title{
Relativistic beaming and the intrinsic properties of extragalactic radio jets
}

M. H. Cohen*

M. L. Lister ${ }^{\dagger}$

D. C. Homan

M. Kadler**

Y. Y. Kovalev

K. I. Kellermann ${ }^{\dagger \dagger}$

R. C. Vermeulen ${ }^{\S}$

his paper is posted at Purdue e-Pubs.

http://docs.lib.purdue.edu/physics_articles/645 
The Astrophysical Journal, 658:232-244, 2007 March 20

(C) 2007. The American Astronomical Society. All rights reserved. Printed in U.S.A.

\title{
RELATIVISTIC BEAMING AND THE INTRINSIC PROPERTIES OF EXTRAGALACTIC RADIO JETS

\author{
M. H. Cohen, ${ }^{1}$ M. L. Lister, ${ }^{2}$ D. C. Homan, ${ }^{3}$ M. Kadler, ${ }^{4,5}$ K. I. Kellermann, ${ }^{6}$ \\ Y. Y. Kovalev, ${ }^{5,7,8}$ and R. C. Vermeulen ${ }^{9}$ \\ Received 2006 October 6; accepted 2006 November 16
}

\begin{abstract}
Relations between the observed quantities for a beamed radio jet, which are the apparent transverse speed and the apparent luminosity $\left(\beta_{\mathrm{app}}, L\right)$, and the intrinsic quantities, which are the Lorentz factor and the intrinsic luminosity $(\gamma$, $L_{0}$ ), are investigated. The inversion from measured to intrinsic values is not unique, but approximate limits to $\gamma$ and $L_{0}$ can be found using probability arguments. Roughly half the sources in a flux density-limited, beamed sample have a

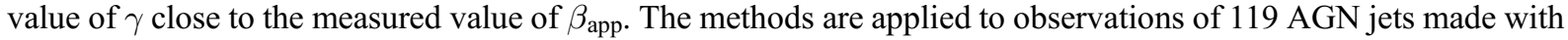
the VLBA at $15 \mathrm{GHz}$ during 1994-2002. The results strongly support the common relativistic beam model for an extragalactic radio jet. The $\left(\beta_{\text {app }}, L\right)$ data are closely bounded by a theoretical envelope, an "aspect" curve for $\gamma=32$ and $L_{0}=10^{25} \mathrm{~W} \mathrm{~Hz}^{-1}$. This gives limits to the maximum values of $\gamma$ and $L_{0}$ in the sample: $\gamma_{\max } \approx 32$, and $L_{0, \max } \sim 10^{26} \mathrm{~W} \mathrm{~Hz}^{-1}$. No sources with both high values of $\beta_{\text {app }}$ and low values of $L$ are observed. This is not the result of selection effects due to the observing limits, which are a flux density of $S>0.5 \mathrm{Jy}$ and an angular velocity of

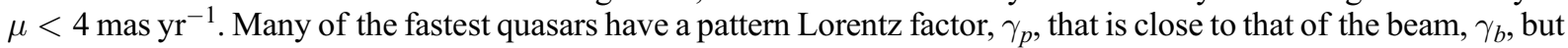
some of the slow quasars must have $\gamma_{p} \ll \gamma_{b}$. Three of the 10 galaxies in the sample have a superluminal feature, with speeds up to $\beta_{\text {app }} \approx 6$. The others are at most mildly relativistic. The galaxies are not off-axis versions of the powerful quasars, but Cygnus A might be an exception.
\end{abstract}

Subject headings: BL Lacertae objects: general — galaxies: active — galaxies: individual (Cygnus A) galaxies: jets — galaxies: statistics — quasars: general

\section{INTRODUCTION}

In recent years, VLBI observations have provided many accurate values of the apparent luminosity, $L$, of compact radio jets and of the apparent transverse speed, $\beta_{\text {app }}$, of features (components) moving along the jets. These quantities are of considerable interest, but the intrinsic physical parameters, which are the Lorentz factor, $\gamma$, and the intrinsic luminosity, $L_{0}$, are more fundamental. In this paper we first consider the "inversion problem"; that is, the estimation of intrinsic quantities from observed quantities. We then apply the results to data from a large multiepoch survey that we have carried out with the VLBA at $15 \mathrm{GHz}$.

The inversion problem is discussed in $\S \S 2-4$ with an idealized relativistic beam, one that has the same vector velocity everywhere and contains a component moving with the beam velocity. The jet emission is Doppler boosted, and Monte Carlo simulations are used to estimate the probabilities associated with selecting a source: that of selecting $\left(\beta_{\text {app }}, L\right)$ from a given $\left(\gamma, L_{0}\right)$, and the converse, the probability of $\left(\gamma, L_{0}\right)$ being the intrinsic parameters for an observed $\left(\beta_{\text {app }}, L\right)$.

\footnotetext{
${ }^{1}$ Department of Astronomy, California Institute of Technology, Pasadena, CA; mhc@astro.caltech.edu.

2 Department of Physics, Purdue University, West Lafayette, IN; mlister@ physics.purdue.edu.

${ }^{3}$ Department of Physics and Astronomy, Denison University, Granville, OH; homand@denison.edu.

${ }^{4}$ Astrophysics Science Division, NASA Goddard Space Flight Center, Greenbelt, MD; mkadler@milkyway.gsfc.nasa.gov.

5 Max-Planck-Institut für Radioastronomie, Bonn, Germany; ykovalev@, mpifr-bonn.mpg.de.

${ }^{6}$ National Radio Astronomy Observatory, Charlottesville, VA; kkellerm@ nrao.edu.

7 Astro Space Center, Lebedev Physical Institute, Moscow, Russia.

8 Jansky Fellow; National Radio Astronomy Observatory, Green Bank, WV.

9 ASTRON, Netherlands Foundation for Research in Astronomy, Dwingeloo, Netherlands; rvermeulen@astron.nl.
}

In $\S 4$ we introduce the concept of an "aspect" curve, defined as the track of a source on the ( $\left.\beta_{\text {app }}, L\right)$-plane (the observation plane) as $\theta$ (the angle to the line of sight [LOS]) is varied, and an "origin" curve, defined as the set of values on the $\left(\gamma, L_{0}\right)$-plane (the intrinsic plane) from which the observed source can be expressed. These provide a ready way to understand the inversion problem and illustrate the lack of a unique inversion for a particular source. Probabilistic limits provide constraints on the intrinsic parameters for an individual source, but when the entire sample of sources is considered, more general comments can be made, as in $\S 7$.

The observational data are discussed in $\S 5$. They are from a $2 \mathrm{~cm}$ Very Long Baseline Array (VLBA) survey, and they have been published in a series of papers: Kellermann et al. (1998, hereafter Paper I), Zensus et al. (2002, hereafter Paper II), Kellermann et al. (2004, hereafter Paper III), Kovalev et al. (2005, hereafter Paper IV), and E. Ros et al. (2007, in preparation). This is a continuing survey, and the speeds are regularly updated using new data; in this paper we include results up to 2006 September 15. The analysis also includes some results from the MOJAVE program, which is an extension of the $2 \mathrm{~cm}$ survey using a statistically complete sample (Lister \& Homan 2005). Prior to Paper III, the largest compilation of internal motions was in Vermeulen \& Cohen (1994, hereafter VC94), who tabulated the internal proper motion, $\mu$, for 66 active galactic nuclei (AGNs), and the value of $\beta_{\text {app }}$ for all but the 2 AGNs without a redshift. The data came from many observers, using various wavelengths and different VLBI arrays, and consequently were inhomogeneous. The $2 \mathrm{~cm}$ data used here were obtained with the VLBA over the period 1994-2002 and comprise "Excellent" or "Good" apparent speeds (see Paper III) for components in 119 sources. This is a substantial improvement over earlier data sets, and it allows us to make statistical studies that previously have not been possible. Other recent surveys are reported by Jorstad et al. (2005, hereafter J05), with data on 15 AGNs at $43 \mathrm{GHz}$; by Homan et al. (2001), with 
data on 12 AGNs at 15 and $22 \mathrm{GHz}$; and by Piner et al. (2006), with data on $77 \mathrm{AGNs}$ at $8 \mathrm{GHz}$.

In some sources it is clear that the beam and pattern speeds are different, and to discuss this we differentiate between the Lorentz factor of the beam, $\gamma_{b}$, and that of the pattern, $\gamma_{p}$. In most of this paper, however, we assume that $\gamma_{b} \approx \gamma_{p}$ and drop the subscripts. In $\S \S 6$ and 7, peak values for the distributions of $\gamma$ and $L_{0}$ in the sample are discussed, and in $\S 8$ the low-velocity quasars and BL Lac objects are discussed. It is likely that some of these have components whose pattern speed is significantly less than the beam speed. The radio galaxies in our sample are discussed in $\S 9$, and we show that most of them are not high-angle versions of the powerful quasars. Cygnus A may be an exception, and we speculate that it contains a fast central jet (a spine), with a slow outer sheath. In this paper we use a cosmology with $H_{0}=70 \mathrm{~km} \mathrm{~s}^{-1}$ $\mathrm{Mpc}^{-1}, \Omega_{m}=0.3$, and $\Omega_{\Lambda}=0.7$.

\section{RELATIVISTIC BEAMS}

In this section the standard relations for an ideal relativistic beam (e.g., Blandford \& Königl 1979) are reviewed. The beam is characterized by its Lorentz factor, $\gamma$, its intrinsic luminosity, $L_{0}$, and its angle to the line of sight, $\theta$. From these, the Doppler factor, $\delta$, the apparent transverse speed, $\beta_{\text {app }}$, and the apparent luminosity, $L$, can be calculated:

$$
\begin{gathered}
\delta=\gamma^{-1}(1-\beta \cos \theta)^{-1}, \\
\beta_{\text {app }}=\frac{\beta \sin \theta}{1-\beta \cos \theta}, \\
L=L_{0} \delta^{n}
\end{gathered}
$$

where $\beta=\left(1-\gamma^{-2}\right)^{1 / 2}$ is the speed of the beam in the AGN frame (units of $c$ ) and $L_{0}$ is the luminosity that would be measured by an observer in the frame of the radiating material. The exponent $n$ in equation (3) combines effects due to the $K$-correction and those due to Doppler boosting: $n=\alpha+p$, where $\alpha$ is the spectral index $\left(S \sim \nu^{\alpha}\right)$ and $p$ is the Doppler boost exponent, as discussed in $\S 2.1$.

From equations (1) and (2), any two of the four parameters $\beta_{\text {app }}, \gamma, \delta$, and $\theta$ can be used to find the others; a convenient relation is $\beta_{\text {app }}=\beta \gamma \delta \sin \theta$. Figure $1 a$ shows $\delta$ and $\beta_{\text {app }}$ as functions of $\sin \theta$, all normalized by $\gamma$; the curves are valid for $\gamma^{2} \gg 1$. When $\sin \theta=\gamma^{-1}, \delta=\gamma$ and $\beta_{\text {app }}=\beta_{\text {app, } \max }=\beta \gamma$. The "critical" angle $\theta_{c}$ is defined by $\sin \theta_{c}=\gamma^{-1}$, and the approximation $\theta / \theta_{c} \approx \gamma \sin \theta$ will be used; this is accurate for $\gamma^{2} \gg 1$ and $\theta^{2} \ll 1$ and is correct to $20 \%$ for $\theta<60^{\circ}$ and $\beta>0.5$.

It is also useful to regard $\beta_{\text {app }}$ and $\theta$ as the independent quantities. Figure $1 b$ shows $\delta$ and $\gamma$ as functions of $\sin \theta$, all normal-

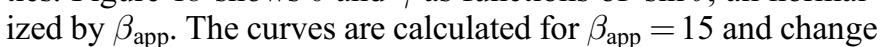
slowly with $\beta_{\text {app }}$ for $\beta_{\text {app }}^{2} \gg 1$.

\subsection{Assumptions}

In our analysis we assume that a source contains an ideal relativistic beam: one that is straight and narrow, and in which the pattern speed is the same as the beam speed: $\gamma_{p} \approx \gamma_{b}$. In particular, the Doppler factor for the core must be derived from the same values of $\gamma$ and $\theta$ that apply to the value of $\beta_{\text {app }}$ for the moving component, which is several pc or more away. Many sources, however, are seen to have more than one moving component, and they may have different values of $\beta_{\text {app. In these cases we }}$ have selected the fastest speed, on the grounds that of all the components, it is the one most likely to be moving at nearly the beam speed. It probably is due to a shock associated with an outburst in flux density, while some of the slower components might be trailing shocks (Agudo et al. 2001). The main shock itself must be moving faster than the beam, but the synchrotron source, which is a density concentration behind the shock, can have a net speed slower than the shock, as shown by numerical simulations (Agudo et al. 2001).

In some sources the only component we see is a stationary one at a bend in the jet. We believe that in these cases we see a standing shock, or perhaps enhanced radiation from a section of the jet that is tangent to the LOS. These components will have $\gamma_{p} \ll \gamma_{b}$ and might be part of the population of slow quasars discussed in $\S 8$.

It is clear that some jets are not straight and that $\theta$ is not the same in the core and in the moving components. See, for example, 3C 279 (Homan et al. 2003), where the velocity vector changed during the course of observations, and $0735+178$ and $2251+158$, where the image shows a jet with sharp bends (Paper I). However, in cases in which at least moderate superluminal motion is found, the motion must be close to the LOS, and any changes in angle will be strongly amplified by projection. An observed right-angle bend could correspond to an intrinsic bend of only a few degrees.

The Doppler boost exponent $p$ depends on geometry and optical depth and is discussed by Lind \& Blandford (1985). For a smooth jet, $p=2$, and this value is appropriate for the core region, where, we assume, the relativistic beam streams through a stationary $\tau=1$ region. In some cases the moving component can be modeled as an isolated optically thin source, which would have $p=3$, but for most of the sources the flux density is dominated by radiation from the core, and we use $p=2$ here. See Figure 5 in Paper IV. The other term in the exponent $n$ is the spectral index $\alpha$. Nearly all the sources have a "flat" spectrum, with $|\alpha|<0.5$, and they also have variable flux density and a variable spectrum. Because of the time dependence, it is not possible to generate a useful index for each source, and we take $\alpha=0$ as a rough global average, giving $n=2$. This is further justified in $\S 5.3$, where it is shown that $n=3$ does not fit the data. However, this choice of $\alpha=0$ clearly leads to errors for those sources with a high Doppler factor, say, $\delta=30$, because the $K$-correction must cover a frequency range of a factor of 30 . This introduces uncertainty into the estimates of the intrinsic luminosity.

We shall use equation (3) as if $L_{0}$ were independent of $\theta$, but this is not necessarily so. The opacity in the surrounding material may change with $\theta$, and the luminosity of any optically thick component may change with angle. Other changes in $L_{0}$ might be caused by a change in location of the emission region when $\theta$, and therefore the Doppler factor and the emission frequency, changes (Lobanov 1998).

\section{PROBABILITY}

The probability of selecting a source with a particular value of $\theta, \gamma, \beta_{\text {app }}$, or $\delta$ from a flux density-limited sample of relativistically boosted sources is central to our discussion. Because $S \propto \delta^{2}$ ( $\S 5.3$ ) and $\delta$ decreases with increasing $\theta$, the sources found will preferentially be at small angles, even though there is not much solid angle there. VC94 calculated the probability $p\left(\theta \mid \gamma_{f}\right)$ (the subscript $f$ means "fixed") in a Euclidean universe, and Lister \& Marscher (1997, hereafter LM97) extended this with Monte Carlo calculations to include evolution. However, the observations directly give $\beta_{\mathrm{app}}$, not $\gamma$, and $p\left(\theta \mid \beta_{\mathrm{app}, f}\right)$ is generally not an analytic function. To deal with this, M. Lister et al. (2007, in preparation) use Monte Carlo methods to study the probability functions. We use one of their simulations here as an illustrative example.

In the Monte Carlo calculation, a simulated parent population is created (see the Appendix), from which 100,000 sources 

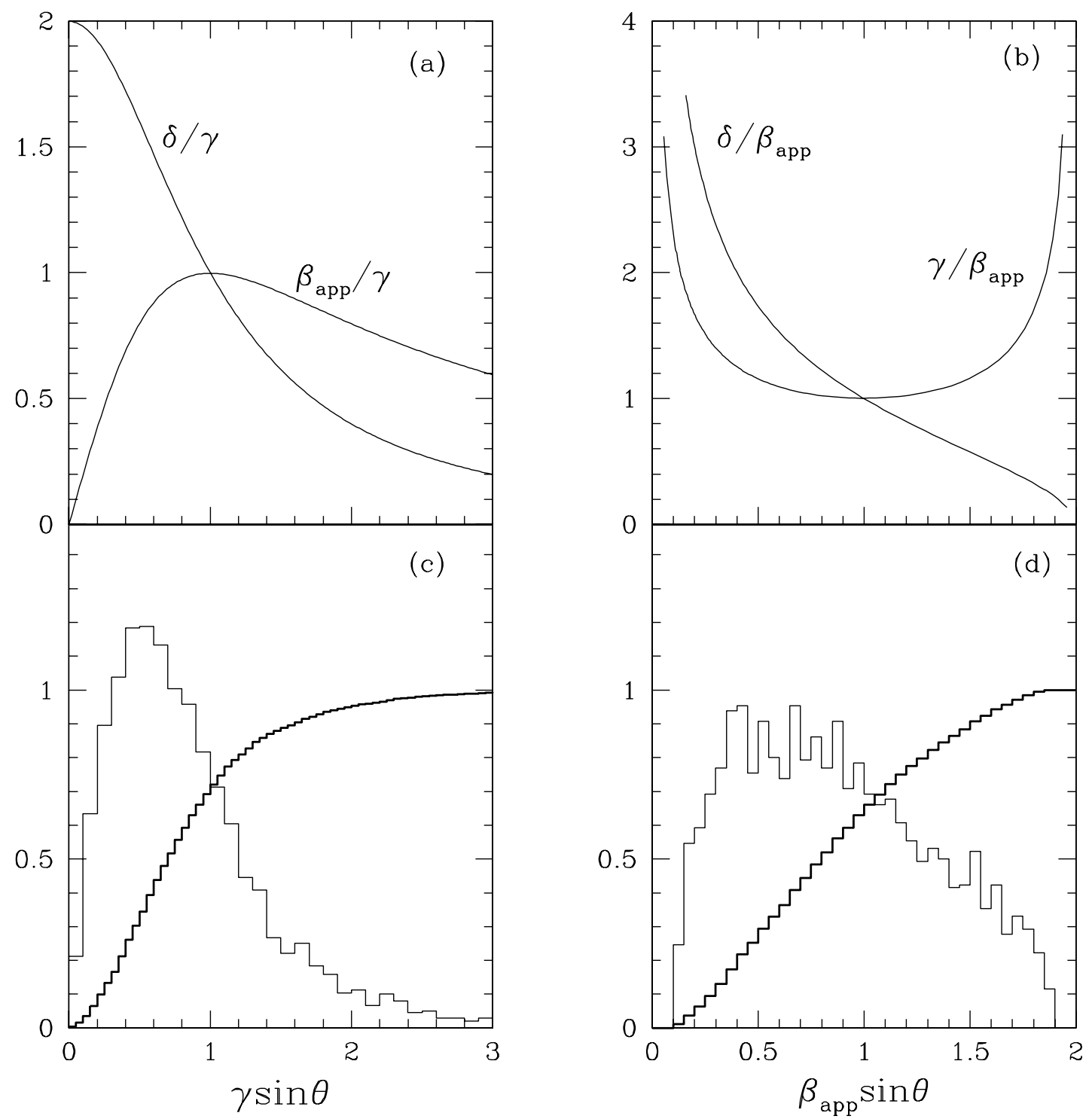

FIG. 1. - Top: Parameters for a relativistic beam with a Lorentz factor of $\gamma$ and an angle to the LOS of $\theta$. (a) Curves are plotted for $\gamma=15$ but change slowly with $\gamma$, provided that $\gamma^{2} \gg 1$. (b) Curves are plotted for $\beta_{\text {app }}=15$ and change slowly with $\beta_{\text {app }}$, provided that $\beta_{\text {app }}^{2} \gg 1$. In $(a)$ the quantities are normalized by the constant Lorentz factor; in $(b)$, they are normalized by the constant apparent speed. Bottom: Results from a Monte Carlo simulation of a flux density-limited survey selected from the parent population described in the Appendix. (c) Probability density $p\left(\theta \mid \gamma_{f}\right)$ and cumulative probability $P\left(\theta \mid \gamma_{f}\right)$ (heavy line) for $\gamma \approx 15$. Roughly $75 \%$ of the selected sources will have $\gamma \sin \theta<1$; that is, $\theta<\theta_{c}$. Values of $\gamma \sin \theta<0.15$ and $>2.0$ are unlikely; the cumulative probabilities are approximately 0.04 and 0.96 , respectively. (d) Probability density and cumulative probability for selecting a source at angle $\theta$, for $\beta_{\text {app }} \approx 15$. As $\beta_{\text {app }}$ decreases, the probability curve becomes more peaked, and the peak moves to the left.

with $S>1.5$ Jy are drawn. We select a slice of this sample with $14.5 \leq \gamma \leq 15.5$ and form the histograms in Figure $1 c$, showing the probability density $p\left(\theta \mid \gamma_{f}\right)$ and the cumulative probability $P\left(\theta \mid \gamma_{f}\right)$ for those sources with $\gamma \approx 15$. The histograms vary slowly with $\gamma$, provided that $\gamma^{2} \gg 1$. They are similar to the equivalent diagrams calculated by VC94 (their Fig. 7) and by LM97 (their Fig. 5). Figure $1 c$ may be directly compared with Figure $1 a$, which is a purely geometric result from equations (1) and (2). The peak of the probability is at $\sin \theta \approx 0.6 / \gamma$, where $\beta_{\text {app }} \approx 0.9 \gamma$ and $\delta \approx 1.5 \gamma$. The $50 \%$ point of $P\left(\theta \mid \gamma_{f}\right)$ is at $\gamma \sin \theta \approx 0.7$, giving a median value of $\theta_{\text {med }} \approx 0.7 \gamma^{-1} \approx 0.7 \theta_{c}$.

An interesting measure of the cumulative probability is $P\left(\theta=\theta_{c}\right)$, the fraction of the sample lying inside the critical angle. The slow variation of this fraction with $\gamma$ is seen in Figure $2 a$; a rough value is 0.75 ; that is, most beamed sources will be inside their " $1 / \gamma$ cones." In this paper we take $0.04<P<0.96$ as a practical range for the probability. This corresponds, approximately, to $0.15<$ $\theta / \theta_{c}<2$ for $\gamma=15$, and the angular range for this probability range varies slowly with $\gamma$. Figure 9 (in the Appendix) shows the $(\gamma, \theta)$ distribution for 14,000 sources from the simulation, along with the $4 \%$ and $96 \%$ limits.

We have now described $p\left(\theta \mid \gamma_{f}\right)$, the probability for selecting a jet at angle $\theta$ if it has a Lorentz factor $\gamma_{f}$. However, given that we observe $\beta_{\text {app }}$ and not $\gamma$, we must consider also the probability $p\left(\theta \mid \beta_{\mathrm{app}, f}\right)$; that is, the probability of finding a jet at the angle $\theta$ if it has a fixed value of $\beta$ app. We again use a slice of the Monte Carlo simulation, now for $14.5<\beta_{\text {app }}<15.5$, to get the probabilities shown in Figure $1 d$. The probability density curve is broad, and as $\beta_{\text {app }}$ decreases it becomes more peaked. The median value of $\beta_{\text {app }} \sin \theta$ is shown with the dashed line in Figure $2 b$, as a function of $\beta_{\text {app. }}$.

The probability $p\left(\gamma \mid \beta_{\mathrm{app}, f}\right)$ is also of interest. Figure $3 a$ shows an example, for $\beta_{\text {app }} \approx 15$. The probability is sharply peaked at $\gamma \sim \beta_{\text {app }}$. The median value is $\gamma_{\text {med }} / \beta_{\text {app }}=1.08$, and it changes 

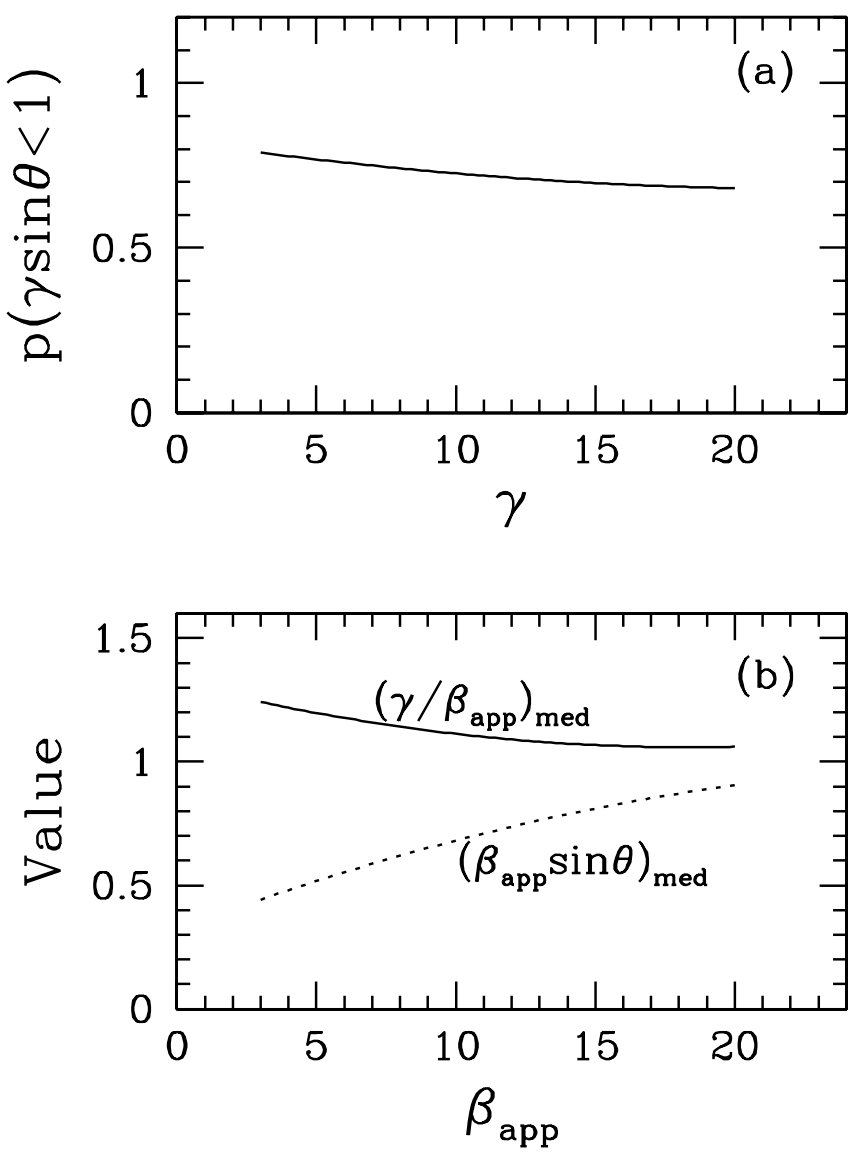

Fig. 2.-Calculations from the simulation described in the Appendix. (a) Probability that $\gamma \sin \theta<1$, as a function of $\gamma$. (b) Median value of $\gamma / \beta_{\text {app }}$ (solid curve) and median value of $\beta_{\text {app }} \sin \theta$ (dashed curve), as functions of $\beta_{\text {app. }}$.

with $\beta_{\text {app }}$ as shown with the solid line in Figure $2 b$. The sharp peak can be understood in geometric terms. In Figure $1 b$ one sees that there is a large range of $\theta$ over which $\gamma$ changes little from its minimum value near $\beta_{\text {app }}$, and Figure $1 d$ shows that most of the probability is in this range. For about half the sources with $\beta_{\text {app }} \approx$ 15 , the value of $\gamma$ is between 15 and 16 , but the other half is distributed to $\gamma=32$, as shown in Figure 3a. For lack of better information, it often is assumed in the literature that $\gamma \approx \beta_{\text {app }}$, but this is not always valid.

Figure $3 b$ shows $p\left(\delta \mid \beta_{\text {app }, f}\right)$ and $P\left(\delta \mid \beta_{\text {app }, f}\right)$ for $\beta_{\text {app }} \approx 15$. The curves change slowly for $\beta_{\text {app }}^{2} \gg 1$. Unlike the Lorentz factor, the probability for the Doppler factor does not have a sharp peak. Consequently $L_{0}$, which varies as $\delta^{2}$, is poorly constrained by $\beta_{\text {app. }}$.

In this paper a particular Monte Carlo simulation is used to show probability curves in Figures 1 and 3 and, numerically, to find the $4 \%, 50 \%$, and $96 \%$ levels of the cumulative probability distributions. These are fairly robust with regard to evolution and parent luminosity functions. We have compared them to several of the simulations calculated by M. Lister et al. (2007, in preparation), and the variations are not large enough to materially affect any of the conclusions in this paper.

Figures $1-3$ are not valid in the nonrelativistic case, in which $\beta^{2} \ll 1, \gamma \approx 1, \delta \approx 1, \beta_{\text {app }} \approx \beta \sin \theta$, and $p(\theta) \sim \sin \theta$. Our discussion is also not valid for samples selected on the basis of nonbeamed emission.

\section{THE INVERSION PROBLEM}

VLBA observations can directly give the apparent speed $\beta_{\text {app }}$ and the apparent luminosity $L$, but the Lorentz factor $\gamma$ and the

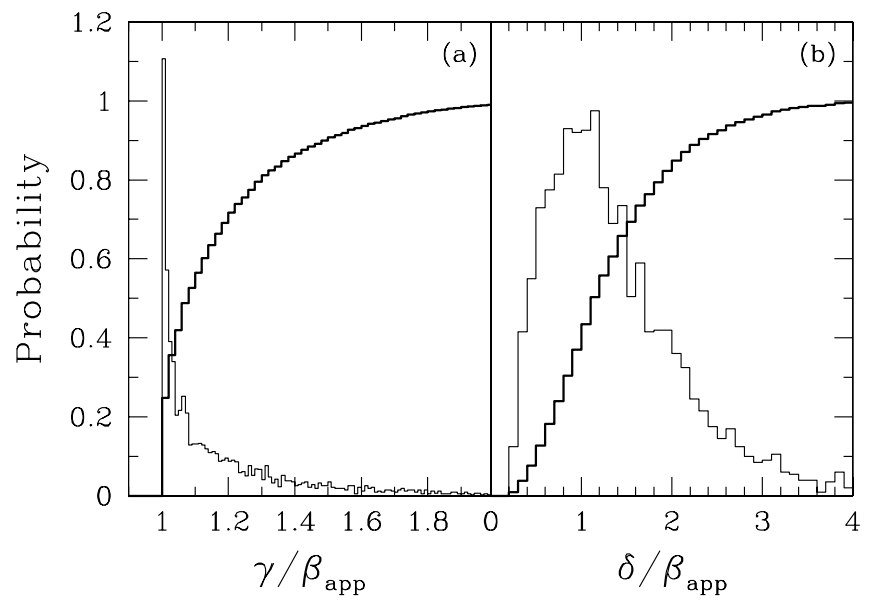

FIG. 3.-Probability density and cumulative probability (heavy line) when $\beta_{\text {app }} \approx 15 .(a) p\left(\gamma \mid \beta_{\mathrm{app}, f}\right) ;(b) p\left(\delta \mid \beta_{\mathrm{app}, f}\right)$.

intrinsic luminosity $L_{0}$ are more useful. We refer to the estimation of the latter from the former as the inversion problem.

The inversion is illustrated with Figure 4. On the left is the intrinsic plane, with axes $\gamma$ and $L_{0}$, and on the right is the observation plane, with axes $\beta_{\text {app }}$ and $L$. Consider a source at point a in Figure $4 a$, with $\gamma=20$ and $L_{0}=2 \times 10^{24} \mathrm{~W} \mathrm{~Hz}^{-1}$. Let it be observed at $\theta=1.3^{\circ}$, so that $\beta_{\text {app }}=15.0$ and $L=2.2 \times 10^{27} \mathrm{~W} \mathrm{~Hz}^{-1}$. This is the point $\mathrm{z}$ in Figure $4 b$. Now let $\theta$ vary, and the observables for source a will follow curve A. We call A an aspect curve. It shows all possible observable $\left(\beta_{\text {app }}, L\right)$ pairs for the given source a. The aspect curve is parametric in $\theta$, with $\theta=0^{\circ}$ on the right, as shown. The height of the curve is fixed by the value of $\gamma$, and the location on the $x$-axis is fixed by $\gamma$ and $L_{0}$. The width of the peak is controlled by the exponent $n$ in equation (3), as discussed in $\S 5.3$.

Now consider a source with observational parameters at point $\mathrm{z}$ in Figure $4 b$. What can be said about the intrinsic parameters for this source? From equations (1)-(3), curve Z in Figure $4 a$ can be drawn; curve $Z$ contains all possible pairs of intrinsic parameters from which source $z$ can be expressed. We call curve $Z$ an origin curve. It is parametric in $\theta$, with $\theta=0^{\circ}$ on the left, as shown. The curve has been truncated at $\gamma=32$, because this is the approximate upper limit of $\gamma$ for our data, as shown in $\S 6$.

Given the lack of a constraint on $\theta$, the inversion for the observed point $z$ in Figure $4 b$ is not unique. Any point on the origin curve $\mathrm{Z}$ in Figure $4 a$ could be its counterpart. This gives limits to $\gamma$ and $L_{0}$, but they usually are broad. The limits get tighter when the probability of observing a boosted source is considered, as in the next section $(\S 4.1)$. More general results apply in a statistical sense when a sample of sources is considered.

\subsection{Probability Cutoffs}

The probabilities associated with observing beamed sources were discussed in $\S 3$. We now use the $4 \%$ and $96 \%$ cumulative probability levels to define the regions in which most of the sources will lie. Figures $4 c$ and $4 d$ are the same as Figures $4 a$ and $4 b$, but with the origin curve truncated at $P\left(\gamma \mid \beta_{\mathrm{app}, f}\right)=4 \%$ and $96 \%$ and the aspect curve similarly truncated at $P\left(\theta \mid \gamma_{f}\right)=4 \%$ and $96 \%$. Note that points $\mathrm{g}$ and $\mathrm{h}$ do not correspond to points $\mathrm{u}$ and $\mathrm{v}$. The probabilities can be seen in Figures $1 d$ and $1 c$, respectively, as functions of $\sin \theta$.

The luminosities are double-valued in Figure 4. The probability cutoffs are found by integrating along curves $A$ and $Z$, and not by accumulating values of $\gamma$ or $\beta_{\text {app }}$ along both sides of the 

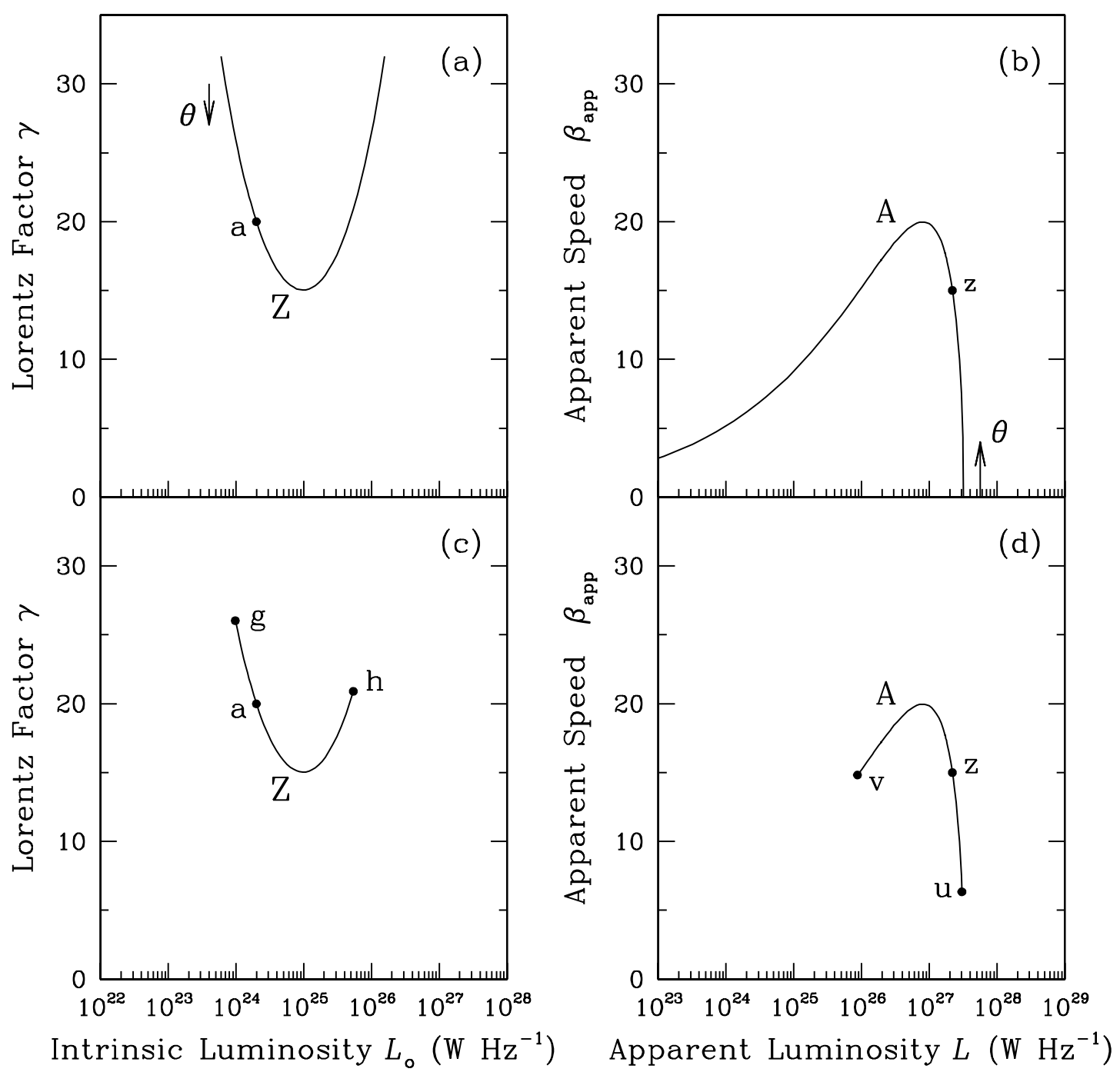

Fig. 4.-Illustrations of the intrinsic (left) and observation (right) planes for relativistic beams. The origin point a in (a), with $\gamma=20$, can be observed anywhere on the aspect curve $\mathrm{A}$ in $(b)$ by varying $\theta$. The observed point $\mathrm{z}$ in $(b)$, with $\beta_{\text {app }}=15$, can be expressed from any point on the origin curve $\mathrm{Z}$ in $(a)$. Both curves are parametric in $\theta$, with $\theta$ increasing as shown. The maximum of the aspect curve in $(b)$ is at $\theta=2.9^{\circ}$ and $\beta_{\text {app }}=19.97$. The minimum of the origin curve in $(a)$ is at $\theta=3.8^{\circ}$ and $\gamma=15.03$. (c) Same as in $(a)$, but with the origin curve truncated at points $g$ and $\mathrm{h}$, the $4 \%$ and $96 \%$ cumulative probability limits, respectively. $(d)$ Same as in (b), but with the aspect curve truncated at points $u$ and $v$, the $4 \%$ and $96 \%$ cumulative probability limits, respectively.

minimum or peak. An example of accumulating $\gamma$ on both sides of the minimum of an origin curve is in Figure $3 a$.

In Figure $4 c$ the points on curve $\mathrm{Z}$ have different Lorentz factors, but all have $\beta_{\text {app }}=15$. The run of $\gamma$ versus $\theta$ along curve $\mathrm{Z}$ is shown in Figure 5, which essentially is a section of the curve in Figure $1 b$. The probability $p\left(\theta \mid \beta_{\text {app }, f}\right)$, shown in Figure $1 d$, varies slowly along this curve and is indicated with the line width.

From Figure $4 c$ we now have probabilistic limits for the intrinsic parameters of the observed source $\mathrm{z}$. Points $\mathrm{g}, \mathrm{h}$, and the minimum give $15<\gamma<25.6$ and $1.0 \times 10^{24} \mathrm{~W} \mathrm{~Hz}^{-1}<L_{0}<5.4 \times$ $10^{25} \mathrm{~W} \mathrm{~Hz}^{-1}$. Note that these values do not describe a closed box on the $\left(\gamma, L_{0}\right)$-plane. Rather, the possible values must lie on curve $Z$. The highest value of $\gamma$ goes with the lowest intrinsic luminosity, the lowest value of $\gamma$ goes with an intermediate luminosity, and the highest luminosity goes with an intermediate value of $\gamma$.

A large survey will likely contain other sources with values of $\beta_{\text {app }}$ near that of source $z$. They will have various luminosities and will form a horizontal band in Figure $4 d$. That group of sources will have a distribution of $\gamma$ with a minimum of $\gamma_{\min } \approx \beta_{\text {app }}$ and a median of $\gamma_{\text {med }} \approx 1.1 \beta_{\text {app }}$, according to Figure $2 b$. This means

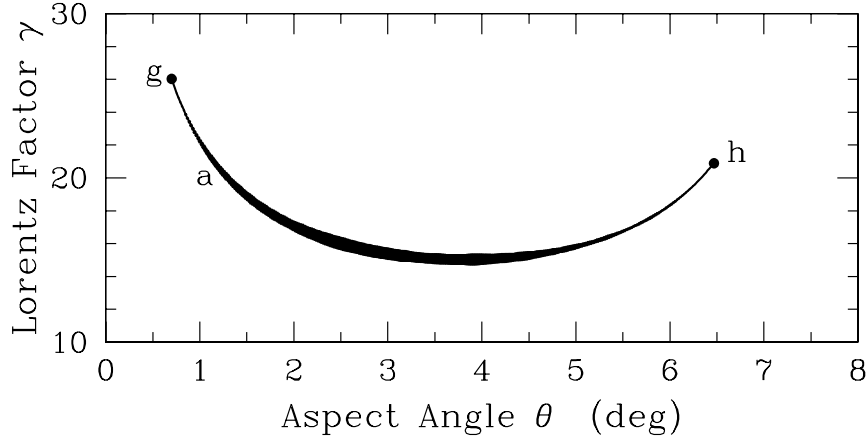

FIG. 5.-Curve $Z$ from Fig. $4 c$, shown in the $(\gamma, \theta)$-plane. The probability density for finding a source with $\beta_{\text {app }} \approx 15$ is indicated by the width of the line. Points $\mathrm{g}$ and $\mathrm{h}$ are the locations at which the cumulative probability $P\left(\theta \mid \beta_{\mathrm{app}, f}\right)$ reaches $4 \%$ and $96 \%$, respectively. For a range of $\theta$ around the maximum probability at $\theta \sim 2.5^{\circ}$, the value of $\gamma$ changes slowly. As is shown more directly in Fig. $3 a$, approximately half the sources with $\beta_{\text {app }}=15$ will have values of $\gamma$ between 15 and 16 . However, $\theta$ is not similarly constrained. 
that, for any individual source, it is reasonable to guess that $\gamma$ is a little larger than $\beta_{\text {app }}$, although that guess will be far off for some of the objects. It is correct to say that about half the survey sources with $\beta_{\text {app }} \approx 15$ will have $15<\gamma<16$ and that about $95 \%$ of them will have $15<\gamma<25.6$. The value of $95 \%$ results from the $4 \%$ that are above point $g$ in Figure $4 c$ and the $1 \%$ that are above $\gamma=25.6$ when the curve is continued above point $\mathrm{h}$.

\section{THE DATA}

The $2 \mathrm{~cm}$ VLBA survey consisted of repeated observations of 225 compact radio sources over the period 1994-2002. Since that time, the MOJAVE program (Lister \& Homan 2005) has continued observing a smaller but statistically complete sample of AGNs. Most of the sources have a "core-jet" structure, with a compact flat-spectrum core at one end of a jet, and with less compact features moving outward, along the jet. The VLBA images were used to find the centroids of the core and the components at each epoch, and a least-squares linear fit was made to the locations of the centroids relative to the core. The apparent transverse velocity was calculated from the angular velocity and the redshift. See Paper III and E. Ros et al. (2007, in preparation) for details.

Each component speed is assigned a quality factor of Excellent, Good, Fair, or Poor according to the criteria presented in Paper III, but only the 127 sources with Excellent or Good components are used here. Eight of the 127 sources are conservatively classified by us as gigahertz-peaked spectrum (GPS) sources. This classification is given only to sources that have always met the GPS spectral criteria given by de Vries et al. (1997) and is based on RATAN monitoring of broadband instantaneous radio spectra of AGNs (Kovalev et al. 1999). In GPS sources the bulk of the radiation is not highly beamed, as it must be if our model is to be applicable, and we omit the GPS sources from this study. The final sample contains 119 sources, comprising 10 galaxies, 17 BL Lac objects, and 92 quasars, as classified by Véron-Cetty \& Véron (2003). (See the classification discussion in Paper IV.) The sample and the $\left(\beta_{\text {app }}, L\right)$ values used here are given on our Web site. ${ }^{10}$ The $\beta_{\text {app }}$ data are updated from values in Paper III, with the addition of results from more recent epochs that are given in E. Ros et al. (2007, in preparation) and on our Web site.

Values of $\left(\beta_{\mathrm{app}}, L\right)$ for the 119 sources are plotted in Figure 6. Error bars are derived from the least-squares fitting routine for the angular velocity. The luminosities are calculated, for each source, from the median value of the "total" VLBA flux densities, over all epochs, as defined in Paper IV. The quantity $S_{\mathrm{VLBA} \text {, med }}$ is the integrated flux density seen by the VLBA, or the fringe visibility amplitude on the shortest VLBA baselines. The luminosity calculation assumes isotropic radiation. Error bars are not shown for the luminosities. Actual errors in the measurement of flux density are no more than 5\% (Paper IV), but most of the sources are variable over time (see Paper IV, their Fig. 11).

An aspect curve for $\gamma=32$ and $L_{0}=10^{25} \mathrm{~W} \mathrm{~Hz}^{-1}$ is shown in Figure 6. It forms a close envelope to the data points for $L>10^{26} \mathrm{~W} \mathrm{~Hz}^{-1}$. At lower luminosity the curve is well above the data, and, as shown in $\S \S 7$ and 8 , lower aspect curves should be used there to form an envelope. A plot similar to the one in Figure 6 is given in Vermeulen (1995) for the early data from the Caltech-Jodrell Bank $6 \mathrm{~cm}$ survey ( Taylor et al. 1996). Although no aspect curve is shown in Vermeulen (1995), it is clear that the general shape of the distribution is similar at 6 and $2 \mathrm{~cm}$. The parameters of the aspect curve in Figure 6 are used in $\S 7$ to derive limits to the distributions of $\gamma$ and $L_{0}$ for the quasars.

\footnotetext{
${ }^{10}$ See also spectra shown on our Web site, http://www.physics.purdue.edu/ astro/MOJAVE/.
}

\subsection{Selection Effects}

A striking feature of Figure 6 is the lack of sources to the left of the aspect curve; that is, we found no high- $\beta_{\text {app }}$, low- $L$ sources. We recognize two possible selection effects that might influence this, which are the lower flux density limit to the survey and the maximum angular velocity that we can detect. We now combine these to derive a limit curve.

The $2 \mathrm{~cm}$ survey includes sources stronger than $1.5 \mathrm{Jy}$ for northern sources and stronger than 2.0 Jy for southern sources (Paper I). Additional sources that did not meet these criteria but were of special interest are also included in the full sample. However, here we use the median VLBA flux density values from Paper IV for the subsample of 119 sources for which we have good quality kinematic data, and the median of these values is $1.3 \mathrm{Jy}$. We choose $S_{\min }=0.5 \mathrm{Jy}$ as the lower level of "detectability," although $10 \%$ of the sources are below this limit. The completeness level is actually higher, probably close to $1.5 \mathrm{Jy}$, but the survey sources form a representative sample of the population of sources with $S_{\mathrm{VLBA}, \text { med }}>0.5 \mathrm{Jy}$.

The angular velocity limit, $\mu_{\max }$, is set by a number of factors, including the complexity and rate of change of the brightness distribution, the fading rate of the moving components, and the interval between observing sessions. These vary widely among the sources, and there is no easily quantified value for $\mu_{\max }$. In practice, we adjusted the observing intervals for each source according to these factors, with $\Delta T$ being about $1 \mathrm{yr}$ in most cases. This was usually sufficient to eliminate any ambiguity in defining the angular velocity as seen on the "speed plots," which are the position versus time plots shown in Figure 1 of Paper III. For some sources there was little or no change in $1 \mathrm{yr}$, and these were then observed less frequently. For others, a 1 yr separation was clearly too long, and they were observed more frequently, typically twice per year for complex sources. The fastest angular speeds we

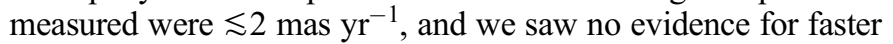
motions that would require more frequent observations. It is important to note that even programs with shorter sampling intervals, down to every 1 or 2 months, have not detected many speeds over $1 \mathrm{mas} \mathrm{yr}^{-1}$, and none significantly larger than $2 \mathrm{mas} \mathrm{yr}^{-1}$ (Gómez et al. 2001; Homan et al. 2001; Jorstad et al. 2005).

A rough limit on our ability to identify very fast components is given by our typical $1 \mathrm{yr}$ observing interval and the fading behavior of jet components. From an analysis of six sources, Homan et al. (2002) found that the flux density of jet components fades with distance from the core as $R^{-1.3}$. If a jet component is first identified at a separation of 0.5 mas with a flux density of $50 \mathrm{mJy}$, that component will probably have faded from view when it is 4 or 5 mas away, where it will have a flux density of only a few mJy. Such a component, appearing just after a set of observations, could fade from view before the next observation a year later, if it were mov-

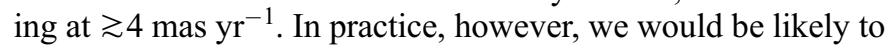
observe such a source in the middle of its cycle, and it would appear to have jet components a few mas from the core that would flicker on and off in an unpredictable fashion. So while we would not have been able to measure the actual speed of such a source, it would have been identified in our sample as unusual and would have been followed up with more frequent observations. Given that we identified no such objects, we take 4 mas $\mathrm{yr}^{-1}$ as a reasonable upper limit to the speeds we are sensitive to with our program.

It is possible that some components could fade more rapidly than the above estimate, and if so, our limit would have to be reduced accordingly. There is some evidence that rapid fading occurs at $43 \mathrm{GHz}$, and in $\S 8$ we describe a source with a component moving more rapidly at $43 \mathrm{GHz}$ than at $15 \mathrm{GHz}$. It is likely that 


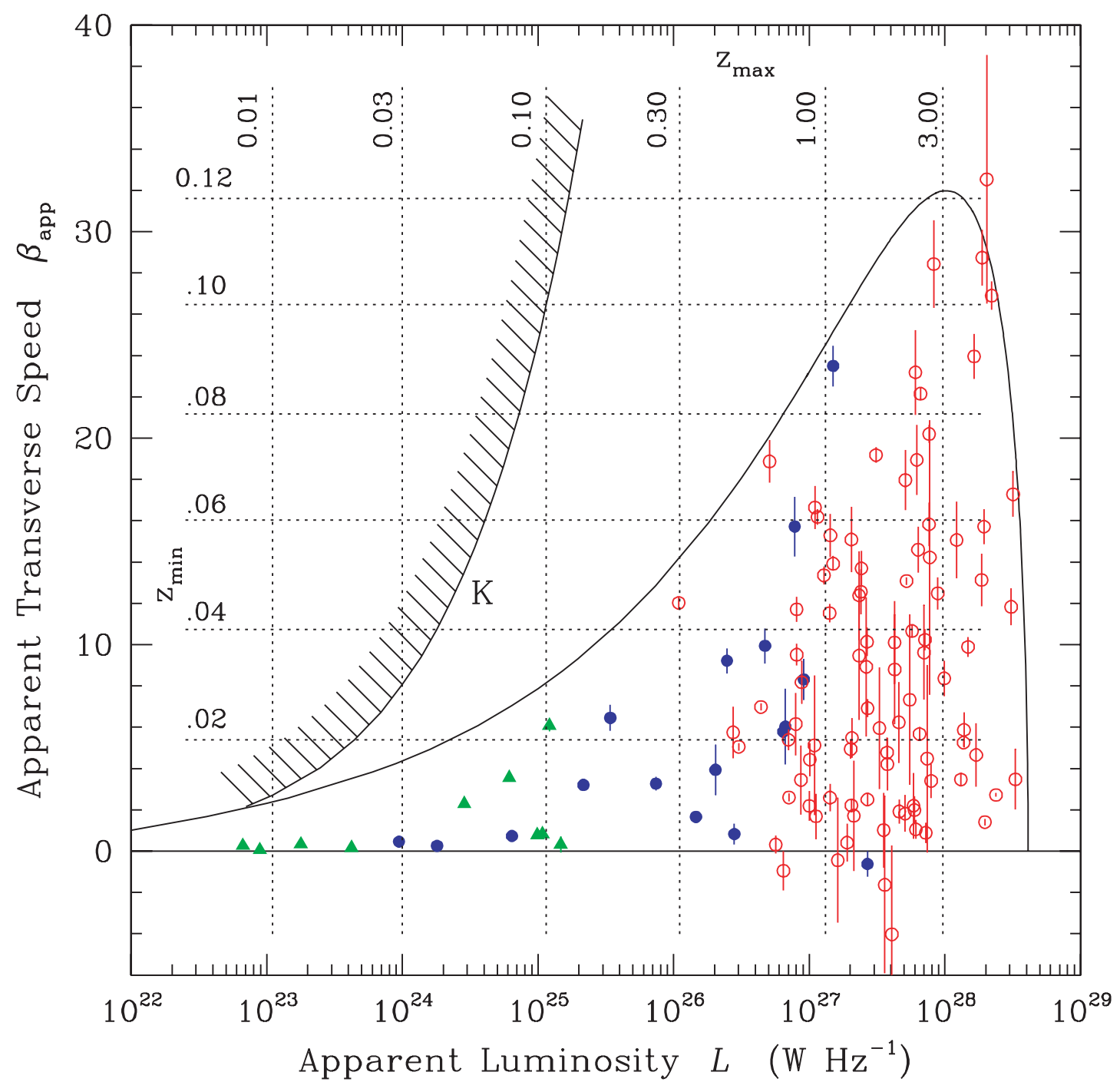

FIG. 6.-Values of apparent transverse speed, $\beta_{\text {app}}$, and apparent luminosity, $L$, for the fastest Excellent or Good component in 119 sources in the $2 \mathrm{~cm}$ VLBA survey. The aspect curve is the locus of $\left(\beta_{\text {app }}, L\right)$ for sources with $\gamma=32$ and $L_{0}=1 \times 10^{25} \mathrm{~W} \mathrm{~Hz}^{-1}$, as $\theta$ varies. Curve K is an observational limit set at $S_{\mathrm{VLBA} \text {,med }}=0.5 \mathrm{Jy}$ and $\mu=4 \mathrm{mas} \mathrm{yr}^{-1}$; the hatched region is usually inaccessible. The horizontal dashed lines indicate the minimum values of redshift, $z_{\min }\left(\beta_{\text {app }}\right)$, for which the angular velocity is below the limit $\mu<4$ mas $\mathrm{yr}^{-1}$. The vertical dashed lines indicate the maximum values of redshift, $z_{\max }(L)$, for which the flux density is above the limit $S>0.5 \mathrm{Jy}$. See $\S 5.1$. Red open circles represent quasars; blue solid circles represent BL Lac objects; green triangles represent galaxies.

the difference is due to a combination of a fast fading rate and better angular resolution, combined with the shorter observing intervals, at $43 \mathrm{GHz}$. Even here, however, the observed speed at $43 \mathrm{GHz}$ is well under the limit.

Curve $\mathrm{K}$ in Figure 6, which is parametric in redshift, is calculated from the limits $S=0.5 \mathrm{Jy}$ and $\mu=4.0 \mathrm{mas} \mathrm{yr}^{-1}$. The hatched region to the left of the curve is inaccessible to our observations, except in special circumstances, such as when the brightness distribution is simple and there is only one feature in the jet. The horizontal dashed lines in Figure 6 show the minimum redshift associated with a value of $\beta_{\mathrm{app}}$, set by the distance at which $\mu=$ 4 mas $\mathrm{yr}^{-1}$, while the vertical dashed lines show the maximum redshift associated with a value of luminosity, set by the distance beyond which the flux density is below $0.5 \mathrm{Jy}$. Thus, every point to the right of curve $\mathrm{K}$ has a range of redshift within which it is observable, and that range fixes a spatial volume. Inspection of the diagram shows that the volume goes to zero at the limit curve and increases toward the envelope. This gradient constitutes the selection effect. Sources are unlikely to be found near the limit curve because the available volume is small. The volume increases toward the envelope; for example, at $L=10^{26} \mathrm{~W} \mathrm{~Hz}^{-1}$ and $\beta_{\text {app }}=20$, the range $0.08 \lesssim z \lesssim 0.30$ is available. In the sample of 119 sources that we use, there are 10 sources in this range, all of which, evidently, are far from the region in question. At $L=$ $10^{25} \mathrm{~W} \mathrm{~Hz}^{-1}$ and $\beta_{\text {app }}=10$, the range $0.04 \lesssim z \lesssim 0.10$ is available, and six of the survey sources are in this range; again, none of them is near the region in question. Hence, the lack of observed sources to the left of the envelope is not a selection effect, but rather must be intrinsic to the objects themselves.

\subsection{The Fast Sources}

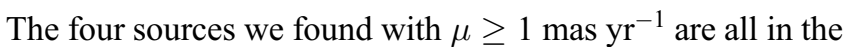
VC94 compilation. VC94 listed four additional sources with $\mu \geq$

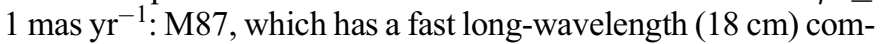
ponent far from the core, Cen A, which is in the southern sky and therefore is not included in our study, and two others, Mrk 421 and $1156+295$, for which our measured values are well below $1 \mathrm{mas} \mathrm{yr}^{-1}$ (Paper III).

We note that, with years of increasingly better observations on more objects, the known number of sources with fast components 


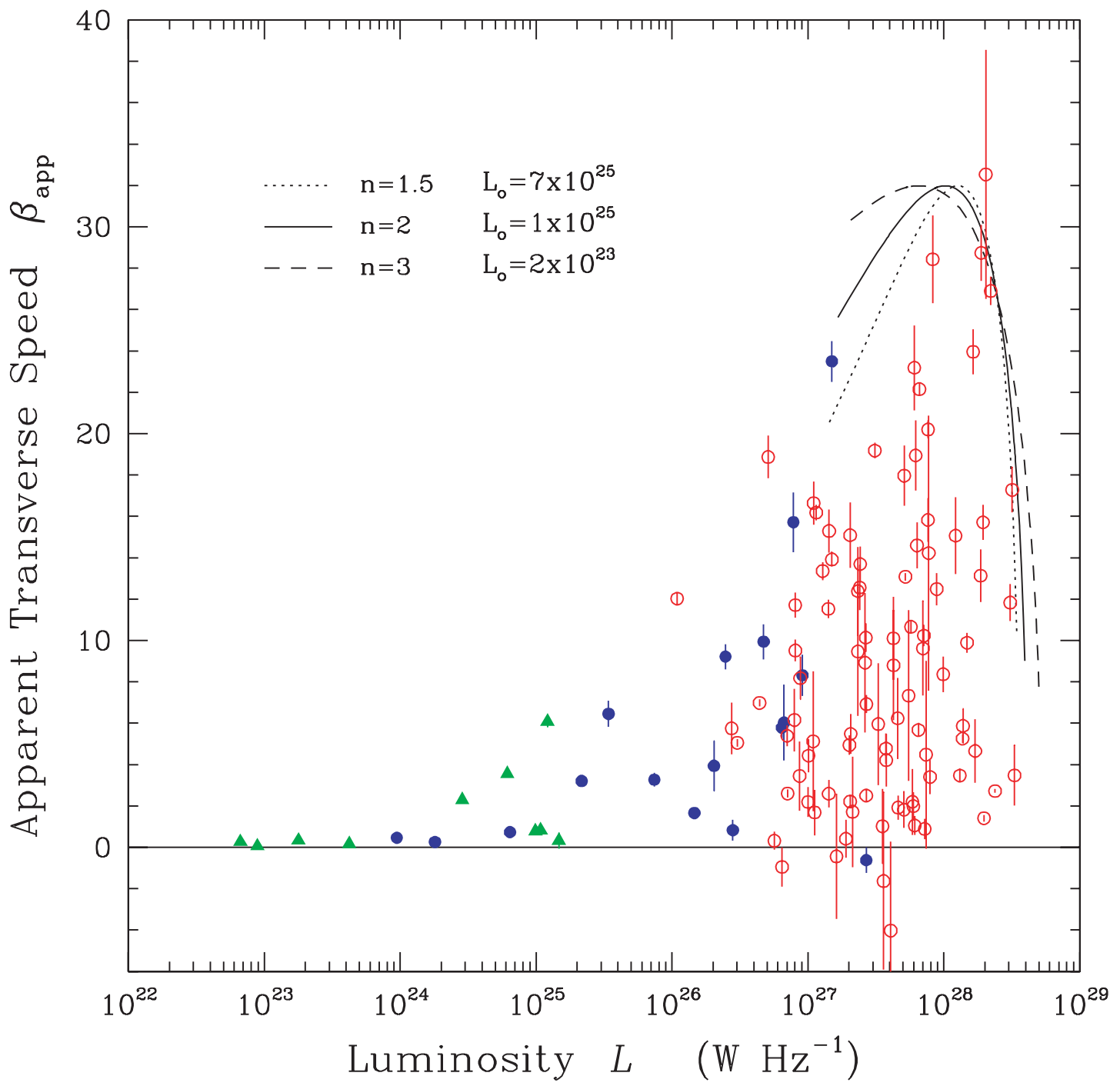

Fig. 7.-As in Fig. 6, but with curves for three values of $n$, the Doppler boost exponent. The curves all have $\gamma=32$ and are truncated at the $4 \%$ and $96 \%$ cumulative probability limits. Values of $L_{0}$ are adjusted to optimize the fit near the top and the right-hand side.

has not increased. There are only five compact jets that show

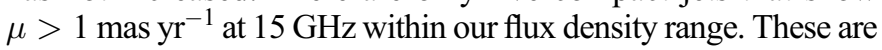
all nearby objects and include three galaxies, 3C 111, 3C 120, and Cen A; one BL Lac object, BL Lac itself; and one quasar, 3C 273. Monthly monitoring at higher resolution by $\mathrm{J} 05$ detected 5 sources (out of 15) that had $\mu>1$ mas $\mathrm{yr}^{-1}$. We found four of these, but

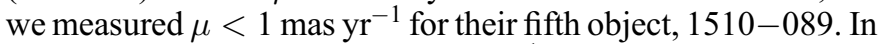

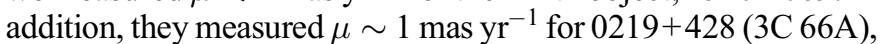
but it has low flux density and is not included in our survey.

\subsection{The Boost Exponent}

The exponent $n$ in equation (3) controls the sharpness of the peak of the aspect curve. Figure 7 shows the data with three aspect curves for $\gamma=32$, with different values of $n$. The curves have been truncated at the $4 \%$ and $96 \%$ probability limits, and the values of $L_{0}$ have been adjusted so that the curves roughly match the right-hand side of the data. The probability was calculated with equation (A15) from VC94, as the simulation described in the Appendix uses $n=2$ and has not been calculated for other values of $n$.

It is important to compare the curves with the data only in the region where the probability is significant. From Figure 7 there is no strong reason to pick one value of $n$ over another. However, if $n=3$, the boosting becomes so strong that strong distant quasars, near the peak of the distribution, have values of $L_{0}$ that are as small as those of the jets in weak nearby galaxies, which (we argue in $\S 9$ ) are only mildly relativistic. This is unrealistic, and we conclude that $n<3$. The value $n=2$ has a theoretical basis ( 2.1 ), and we have adopted it here. Note the large range in intrinsic luminosity corresponding to different values of $n$. Since $n$ is not known with precision, the intrinsic luminosities have a corresponding uncertainty.

\section{THE PEAK LORENTZ FACTOR}

Beaming is a powerful relativistic effect that supplies a strong selection mechanism in high-frequency observations of AGNs. Consider a sample of randomly oriented, relativistically boosted sources that have distributions in redshift, intrinsic luminosity, and Lorentz factor. Make a flux density-limited survey of this sample. VC94 and LM97 have shown that in this case the selected sources will have a maximum value of $\beta_{\mathrm{app}}$ that closely approaches the upper limit of the $\gamma$ distribution, even for a rather small sample size. This comes about because the probability of selecting a source is maximized near $\theta=0.6 \theta_{c}$, where $\beta_{\text {app }} \approx 0.9 \gamma$ (see 


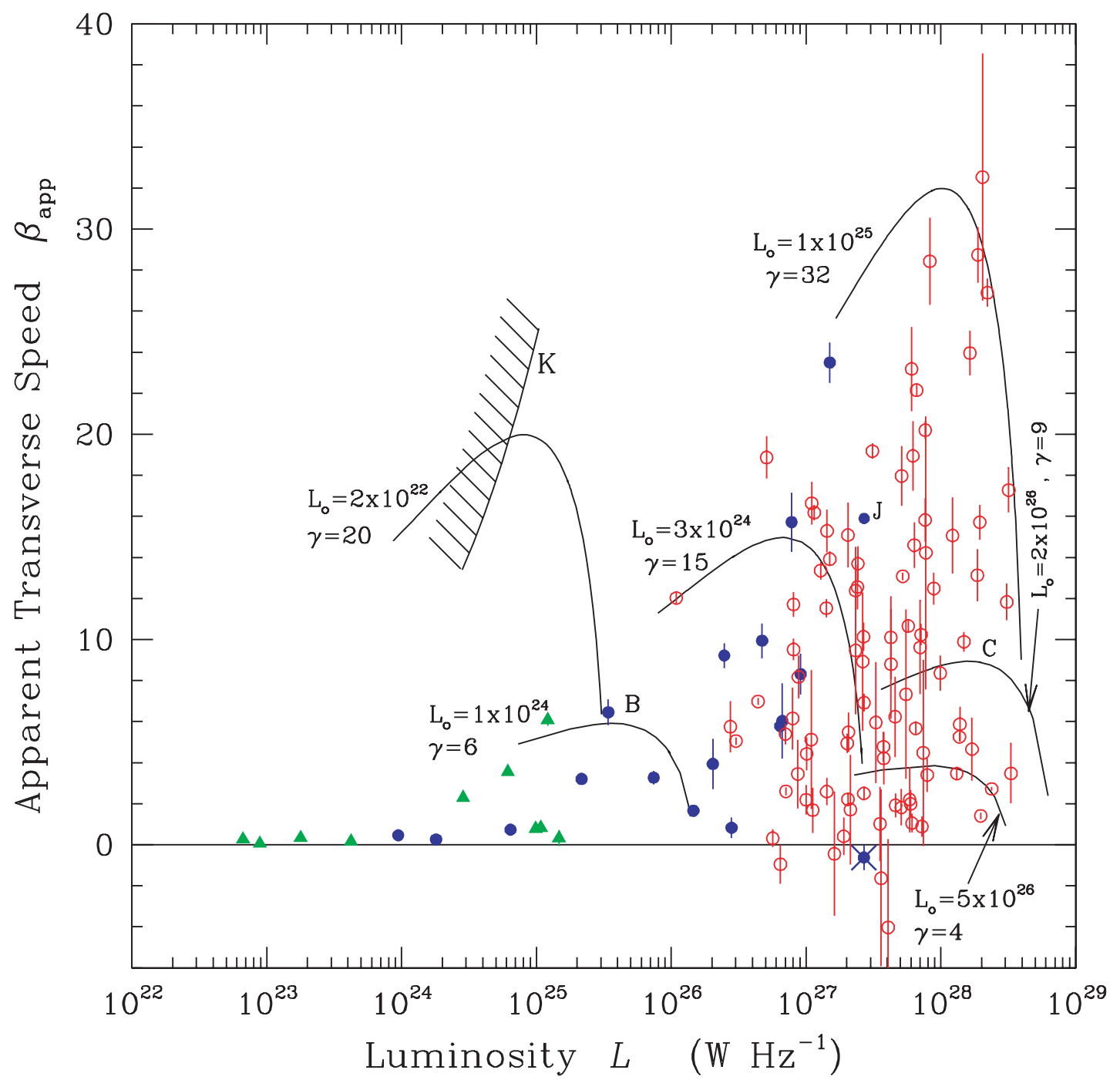

FIG. 8. - As in Fig. 6, but with multiple aspect curves that are truncated at the $4 \%$ and $96 \%$ probability levels. At the peak of each curve, $P(\theta) \approx 0.75$; that is, about $3 / 4$ of the probability of selecting a source with these values of $\gamma$ and $L_{0}$ is on the right side of the curve. The cross close to $\beta_{\text {app }}=0$ marks the source $1803+784$, and point $\mathrm{J}$ is the same source, but with the $\beta_{\text {app }}$ value at $43 \mathrm{GHz}$ from J05; see text. Curve $\mathrm{K}$ is a short section of the limit curve K from Fig. 6 .

Fig. 1c), and there is a high probability that, in a group of sources, some will be at angles close to $\theta_{c}$, where $\beta_{\text {app }} \approx \gamma$. Hence, because $\beta_{\text {app, } \max } \approx 32$, the upper limit of the $\gamma$-distribution is $\gamma_{\text {app,max }} \approx 32$.

\section{THE DISTRIBUTIONS OF $\gamma$ AND $L_{0}$}

We showed in $\S 5.1$ that the lack of sources to the left of the envelope is not a selection effect, but is intrinsic to the objects. Since the envelope is narrow at the top, $\beta_{\text {app }}$ and $L$ are correlated; a high value of $\beta_{\text {app }}$ is found only in sources that also have a high value of $L$, but a low value of $\beta_{\text {app }}$ is found in sources with all values of $L$. This translates into $\gamma$ having a similar correlation with $L_{0}$ for the quasars. The $\gamma$ distribution will be similar to the $\beta_{\text {app }}$ distribution in Figure 7, but flatter: with many points shifted up, but nearly all by less than a factor of 2 above $\beta_{\text {app. }}$. The $L_{0}$ distribution will remain more spread out at low $\gamma$ than at high $\gamma$, leading to the correlation that the highest values of $\gamma$ are found only in jets with high intrinsic luminosity. This is consistent with a result from LM97, which is that Monte Carlo simulations with a negative correlation between $\gamma$ and $L_{0}$ give a poor fit to the statistics of the flux densities from the Caltech-Jodrell Bank survey (Taylor et al. 1996).
The good fit of an aspect curve as an envelope to the data in Figure 6 suggests that the parameters of the curve, $\gamma=32$ and $L_{0}=10^{25} \mathrm{~W} \mathrm{~Hz}^{-1}$, reflect the peak values of $\gamma$ and $L_{0}$ in the population. The distribution of $\gamma$ may be a power law, as suggested by LM97, and, as discussed in $\S 6, \gamma_{\max }=32$ is close to the maximum value in the distribution. We now consider constraints on the peak value for $L_{0}$.

Figure 8 is similar to Figure 6, but with several aspect curves, each showing only the region $0.04<P\left(\theta \mid \gamma_{f}\right)<0.96$. The envelope is now formed by a series of aspect curves with successively lower values of $\gamma$. Most of the sources will have values of $\gamma$ rather close to the values of $\beta_{\text {app }}$, but some will have values of $\gamma$ that are substantially greater (see Figs. $2 b$ and $3 a$ ). In Figure 8 these latter sources will not lie near the top of an aspect curve, but will be further down from the peak. It is more likely that they will be at small angles $\left(\theta<\theta_{c}\right)$ than at large angles.

Consider the BL Lac object marked as point B in Figure 8, near the intersection of the curves with $\gamma=6$ and $\gamma=20$. It could be on either curve, but it is near the low-probability region of the curve with $\gamma=20$. For every source on the curve with $\gamma=20$ that is near the intersection, there should be several farther up the 
TABLE 1

GalaXies with $\beta_{\text {app }}>1$

\begin{tabular}{|c|c|c|c|c|c|c|c|c|}
\hline $\begin{array}{l}\text { IAU Name } \\
\text { (1) }\end{array}$ & $\begin{array}{l}\text { Alias } \\
\text { (2) }\end{array}$ & $\begin{array}{l}\text { Type } \\
\text { (3) }\end{array}$ & $\begin{array}{l}\text { Redshift } \\
\text { (4) }\end{array}$ & $\begin{array}{l}\beta_{\text {app }} \\
\text { (5) }\end{array}$ & $\begin{array}{l}D_{\text {var }} \\
(6)\end{array}$ & $\begin{array}{c}\gamma \\
(7)\end{array}$ & $\begin{array}{c}\theta \\
\text { (deg) } \\
(8)\end{array}$ & $\begin{array}{c}L_{0} \\
\left(\mathrm{~W} \mathrm{~Hz}^{-1}\right) \\
(9)\end{array}$ \\
\hline $0415+379$ & 3C 111 & Sy1 & 0.049 & $6.1 \pm 0.1$ & 3.4 & 7.3 & 15 & $4.4 \times 10^{23}$ \\
\hline $0430+052 \ldots \ldots \ldots \ldots \ldots \ldots$ & $3 \mathrm{C} 120$ & Sy1 & 0.033 & $3.6 \pm 0.2$ & 2.4 & 4.1 & 22 & $4.8 \times 10^{23}$ \\
\hline 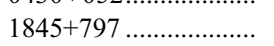 & 3C 390.3 & Sy1 & 0.057 & $2.3 \pm 0.1$ & 0.9 & 3.8 & 42 & $3.1 \times 10^{24}$ \\
\hline
\end{tabular}

curve. Note that the $\gamma=20$ curve intersects the limit curve $\mathrm{K}$ to the left of the peak. There is little available redshift volume at the peak, but the volume increases rapidly at lower values of $\beta_{\text {app}}$, and the lack of sources there means that source B is unlikely to have $\gamma=20$. Alternatively, it could be close to an extension of the curve with $\gamma=15$, but then it is again in a low-probability region. There are a number of sources near the peak of the curve with $\gamma=15$, and point $B$ could be a high-angle version of one of them. But the probability of that is well below 0.04 , and there can be few such sources in the entire sample of 119 sources. We conclude that the galaxies and BL Lac objects on the left side of the distribution $\left(L<3 \times 10^{25} \mathrm{~W} \mathrm{~Hz}^{-1}\right)$, with high confidence, are not off-axis versions of the powerful quasars (the curves with $\gamma=15$ and 32), nor are they high- $\gamma$, low- $L_{0}$ sources (the curve with $\gamma=20$ ).

Point B in Figure 8 is the eponymous object BL Lac (2200+ 420). Denn et al. (2000) studied BL Lac in detail and showed that the jet lies on a helix with axis $\theta=9^{\circ}$ and pitch angle $2^{\circ}$. If $\theta=9^{\circ} \pm 2^{\circ}$ is combined with our value for the apparent transverse speed, $\beta_{\text {app }}=6.6 \pm 0.6$, then $\gamma=7 \pm 1$. This agrees with our conclusion above.

Now consider the sources near point $C$ in Figure 8, at $L=3 \times$ $10^{28} \mathrm{~W} \mathrm{~Hz}^{-1}$ and $\beta_{\text {app }}=9$. They could have $\gamma \approx 9$, but in that case there should be several others down the $\gamma=9$ curve to the right, where most of the probability lies. But there are none there. Any aspect curve with a peak farther to the right is unlikely to represent any of the measured points, and so the curve with $\gamma=9$ is about as far to the right as should be considered. If the sources at point $\mathrm{C}$ are on the curve with $\gamma=9$, then their intrinsic luminosity is an order of magnitude greater than that for the sources near the top of the distribution, the fastest quasars. To avoid a negative correlation between $\gamma$ and $L_{0}$, some of the sources near point C should have values of $\gamma=20$ or more, with the appropriate small values of $\theta$. However, others near the right-hand side of the distribution might well have values of $\gamma \sim 9$ or smaller. This means that the distribution of $L_{0}$ could extend up to $10^{26} \mathrm{~W} \mathrm{~Hz}^{-1}$.

\section{QUASARS AND BL LACERTAE OBJECTS WITH $\beta_{\text {app }}<3$}

Twenty-two of the 92 quasars and 3 of the 13 powerful BL Lac objects in Figure $8\left(L>3 \times 10^{25} \mathrm{~W} \mathrm{~Hz}^{-1}\right)$ have $\beta_{\text {app }}<3$ and have low probability if $\gamma>10$. What are the intrinsic properties of this group? We consider three possibilities. (1) They are high- $\gamma$ sources seen nearly end-on and have $P(\theta)<0.04$. We expect only a few such end-on sources out of a group of 105 . Most of the low-speed quasars cannot be explained this way. (2) They are low- $\gamma$, high- $L_{0}$ sources and have $\gamma \sim 3$. We discussed this above for point $\mathrm{C}$ in Figure 8, with $\beta_{\text {app }}=9$; now we consider values of $\beta_{\text {app }}<3$, and the argument is stronger. Unless the most intrinsically luminous sources have low values of $\gamma$, this option is not viable. (3) A more likely situation is that many of these low- $\beta_{\text {app }}$ components appear to be slow because $\gamma_{p}<\gamma_{b}$.
In support of comment 3 , we note that one of the slow objects, $1803+784$, was also observed by $\mathrm{J} 05$ at $43 \mathrm{GHz}$. They find $\beta_{\text {app }}=$ $15.9 \pm 1.9$, whereas at $15 \mathrm{GHz}$ we found $\beta_{\text {app }}=-0.6 \pm 0.6$. The higher resolution at $43 \mathrm{GHz}$ is crucial in detecting fast components in sources like this, because they are within 1 mas of the core, at or below the resolution limit at $15 \mathrm{GHz}$. In Figure 8 the $15 \mathrm{GHz}$ speed for $1803+784$ is shown with a cross, and the $43 \mathrm{GHz} \beta_{\text {app }}$ value, with the $15 \mathrm{GHz}$ luminosity, is shown with point $\mathrm{J}$. It is likely that we have reported a component speed that is not indicative of the beam speed for $1803+784$.

In Figure 8, source $1803+784$ is in a cluster of objects that, formally, have negative speed. However, they all are within $1 \sigma$ of zero, and their negativity is of little significance. A number of other sources have components with similar formally negative speeds, but in addition, they have a component with a larger positive speed. In this paper we have only used the fastest component in each source.

\section{GALAXIES}

The points in Figure 6 appear to run smoothly from low to high apparent luminosity, suggesting that the different types of objects might be closely related. However, the smoothness is supplied by the BL Lac objects, which connect the galaxies and quasars that otherwise are widely separated in apparent luminosity. In addition, the galaxies all have $z \leq 0.2$, and nearly all the quasars have $z>0.4$. The separation is at least partly the result of our restricted sensitivity, coupled with the luminosity functions. We cannot observe "galaxies" at high redshift because our sensitivity is too low, and we see few "quasars" at low redshift because their local space density is so low. In this section we consider whether the galaxies and quasars form separate classes, or, in particular, whether the galaxies might be high- $\theta$ counterparts of the more luminous sources (Urry \& Padovani 1995).

Three galaxies have superluminal components, and their speeds place them with the lower speed quasars, as seen in Figure 8. These fast galaxies, shown in Table 1, all have broad emission lines and are classified as Seyfert 1 (Sy1) galaxies; they are at low redshift and are highly variable at radio wavelengths. The obscuring torus paradigm for Syl galaxies (Antonucci \& Miller 1985) suggests that they are not at large values of $\theta$, and this is confirmed by the observed values of $\beta_{\text {app }}$, which show that $\theta$ must be less than $\theta_{\max }=2 \arctan \beta_{\text {app }}^{-1} \sim 20^{\circ}-45^{\circ}$. To estimate values of $\gamma, \theta$, and $L_{0}$ for these galaxies, we combine the measured value of $\beta_{\text {app }}$ with a variability Doppler factor, $D_{\text {var }}$, derived from the timescale and strength of variations in flux density (e.g., Cohen et al. 2003). The quantity $D_{\text {var }}$ is given by J05 for $0415+379$ and $0430+052$ and by Lähteenmäki \& Valtaoja (1999) for $1845+797$. We have converted the last value to the cosmology used in this paper and use an intrinsic brightness temperature of $T_{b}=2 \times 10^{11} \mathrm{~K}$. This is a characteristic lower limit for sources in their highest brightness states (Homan et al. 2006), and it should be more appropriate than the canonical equipartition value for variability measurements based 
on flux density outbursts. Note that J05 use a different procedure to calculate $D_{\text {var }}$ and do not assume an intrinsic temperature. The values of $D_{\mathrm{var}}$ are model-dependent, and their reliability is difficult to assess.

The Lorentz factors for the quasars are not estimated in this paper, but from Figure 3 it can be seen that many of the quasars must have Lorentz factors close to their apparent speeds. Thus, from Table 1 and Figure 8, the Lorentz factors of the superluminal galaxies are comparable with those for the slower quasars. Their luminosities, however, do not overlap with those for the quasars, indicating that they are a different population.

Only one of the seven slow galaxies $\left(\beta_{\text {app }}<1\right)$ has a value of $\beta_{\text {app }}$ that is consistent with zero $(2 \sigma)$. The others show definite motions, and several must be at least mildly relativistic, with $\beta_{\text {app }}>0.3$. The galaxies with $\beta_{\text {app }}<0.3$ cannot contain a highly relativistic jet, for that would force $\theta$ to be unacceptably small. For example, if $\beta_{\text {app }}=0.3$ and $\gamma=5$, then $\theta=0.35^{\circ}$ and $\delta=9.9$. This gives $\theta / \theta_{c}=0.03$, which is extremely unlikely for an object with $\gamma=5$ (Fig. 1c). In any event, all proposed galaxy-quasar unifications place the galaxy at a high angle, where the flux density and apparent speed are reduced, but a relativistic beam cannot show $\beta_{\text {app }}=0.3$ at any angle not near $0^{\circ}$ (or $180^{\circ}$ ). Hence, the galaxies are neither low-angle nor high-angle versions of the distant quasars. However, Cygnus A may be an exception, as discussed in the next subsection.

Giovannini et al. (2001) have concluded that most radio galaxies (including Fanaroff-Riley type I galaxies) contain relativistic jets. They assumed that all sources have jets with intrinsic bipolar symmetry and used the measured side-to-side ratio with a correlation between lobe power and intrinsic core power to obtain limits on $\beta$ and $\theta$. Our procedure may be more robust, because each source has a measured value of $\beta_{\text {app }}$ and we do not appeal to symmetry of the lobes.

\subsection{Cygnus $A$}

The galaxy Cygnus A $(z=0.056)$ merits special discussion. The radio lobes are exceptionally powerful, and their luminosity is comparable to that of the most powerful and distant radio galaxies. The jets, however, are weak. Optical polarization studies (Ogle et al. 1997) reveal polarized broad lines and show that Cygnus A is a modest quasar. Bartel et al. (1995) used the frontto-back ratio of the jets of Cygnus A at $6 \mathrm{~cm}$, together with $\beta_{\text {app }}$, to estimate $\theta$. We repeat their analysis with our value for $\beta_{\mathrm{app}}$, $0.83 \pm 0.12$, and obtain $45^{\circ}<\theta<70^{\circ}$. This agrees with other estimates of the angle, including those of Ogle et al. (1997), who found $\theta>46^{\circ}$, and Vestergard \& Barthel (1993), who found $\theta \sim 50^{\circ}-60^{\circ}$. The combination of $\beta_{\text {app }}$ and $\theta$ gives $1.24<\gamma<$ 1.36 and $0.59<\beta<0.68$. Cygnus $A$ is mildly relativistic.

On the other hand, because the lobes in Cygnus $A$ are so powerful, we might have expected that it would have a highly relativistic jet. These contradictory ideas can be reconciled with a two-component beam consisting of a fast spine with a slow sheath, as suggested by numerical simulations (e.g., Agudo et al. 2001). The slow beam that we see has $S_{\text {slow }}=1.5 \mathrm{Jy}$. The fast beam is at a high angle to the LOS and is not seen because it is deboosted, and it must be at least a factor of 10 weaker than the slow beam; that is, $S_{\text {fast }}<0.15 \mathrm{Jy}$. If the fast beam has a Lorentz factor of about 10, then if observed at a small angle, its flux density would be up to a few hundred Jy, far higher than that observed in any other source. But Cygnus $\mathrm{A}$ is much closer than most superluminal sources; if the nearest quasar, 3C 273, were at the distance of Cygnus A, its flux density would be within a factor of 2 of our putative value for Cygnus A. If Cygnus A were at $z \sim 1$ and were pointed near the LOS, it would be a normal quasar, with a radio and optical luminosity somewhat below the median for quasars. It is exceptional only because it is accidentally nearby. This model solves the long-standing problem of the strong lobes combined with the weak core.

In this model the total flux density from Cygnus A varies more slowly with $\theta$ than $\delta^{2}$, the commonly assumed law. If this is correct, and if it applies generally to many other radio sources, then it will affect the usual discussions of the unification of radio sources by aspect. See, for example, Chiaberge et al. (2000), who invoke a two-component model in their article on unification.

\section{DISCUSSION}

The aspect curve in Figure 6 is a good envelope to the quasar data, and this suggests that the relativistic beam model is realistic. The slow rise and rapid fall of the curve is a direct consequence of Doppler boosting combined with time contraction, both of which are relativistic effects. We have used the common assumption that the moving VLBI component is traveling with the beam; that is, $\gamma_{p} \approx \gamma_{b}$. But if this is incorrect-for example, if the beam and pattern speeds are independent, or often are far apart - then it is hard to see why an aspect curve should form an envelope. In particular, if they are independent, then some sources should have high values of $\gamma_{p}$ with low values of $\gamma_{b}$, and this could place them to the left of the envelope in Figure 6. The lack of sources there has been emphasized earlier and is evidence that, for the fastest components in many sources, $\gamma_{p}$ and $\gamma_{b}$ are closely related.

In about one-fourth of the BL Lac objects and quasars, the fastest component appears to be moving slowly $\left(\beta_{\text {app }}<3\right)$. These objects are discussed in $\S 8$, where it is shown, using probability arguments, that most of them cannot be high- $\gamma$ sources with a small value of $\theta$. However, other evidence suggests strongly that most of these objects do have a highly relativistic beam. This evidence consists of rapid variability (e.g., Aller et al. 2003; Teräsranta et al. 2005), high apparent brightness temperature measured both directly with VLBI (Paper IV) and indirectly with interstellar scintillations (Kraus et al. 2003; Lovell et al. 2003), gamma-ray emission (e.g., Dondi \& Ghisellini 1995), and observational effects due to differential Doppler boosting, such as the Laing-Garrington effect (Laing 1988). Many of these sources must have $\gamma_{p} \ll \gamma_{b}$, as discussed in $\S 8$.

\section{CONCLUSIONS}

1. The aspect and origin curves provide a useful way to understand the relations between the intrinsic parameters of a relativistic beam, which are the Lorentz factor $\gamma$ and the intrinsic luminosity $L_{0}$, and the observable parameters, which are the apparent transverse speed $\beta_{\text {app }}$ and the apparent luminosity $L$. Limits to the intrinsic parameters for a given observed source are found on the corresponding origin curve that has been truncated by probability arguments.

2. About half the sources with $\beta_{\text {app }}>4$ that are found in a flux density-limited survey will have a value of $\gamma$ within $20 \%$ of the value of $\beta_{\text {app. }}$.

3 . The $2 \mathrm{~cm}$ VLBA survey has yielded high-quality kinematic data for 119 compact radio jets. When plotted on the observation plane, they are bounded by an aspect curve for $\gamma=32$ and $L_{0}=$ $10^{25} \mathrm{~W} \mathrm{~Hz}^{-1}$ that forms a good envelope to the data at high luminosities. From this, with probability arguments, we find that the peak Lorentz factor in the sample is $\gamma \approx 32$ and the peak intrinsic luminosity is $L_{0} \sim 10^{26} \mathrm{~W} \mathrm{~Hz}^{-1}$.

4. There is an observed correlation between $\beta_{\text {app }}$ and $L$ for the jets in quasars: high values of $\beta_{\text {app }}$ are found only in radio jets with high values of $L$. This implies a similar correlation between $\gamma$ and 
$L_{0}$ : high values of $\gamma$ must preferentially exist in jets with high values of $L_{0}$.

5. The Doppler-boosting exponent $n$ for a typical source in the survey must be less than 3 , or else the highly luminous jets with the fastest superluminal speeds would have intrinsic luminosities comparable to those of the slow, nearby galaxies.

6 . There are too many low-speed $\left(\beta_{\text {app }}<3\right)$ quasars in the sample, according to probability arguments. It is likely that some of them have pattern speeds that are substantially lower than their beam speeds.

7. The galaxies have a distribution of Lorentz factors up to $\gamma=7$; three show superluminal motion, but most are only mildly relativistic. They are not off-axis versions of the powerful quasars. Cygnus A may be an exception, and we suggest that it might have a "spine-sheath" morphology.

8. Our results strongly support the common relativistic beam model for compact extragalactic radio jets. The pattern and beam speeds must be approximately equal for the fastest components in many sources.

We are grateful to the rest of the $2 \mathrm{~cm}$ VLBA Survey Team, who have contributed to the data used in this paper, for their support and advice. We thank Tsvi Piran and Manuel Perucho for helpful discussions, Steven Bloom for commenting on the manuscript, and the referee for helpful comments. The MOJAVE project is supported under National Science Foundation grant AST 04-06923. RATAN-600 observations were supported partly by the NASA JURRISS program (W-19611) and the Russian Foundation for Basic Research (01-02-16812, 02-02-16305, and 05-02-17377). Y. Y. Kovalev is a Research Fellow of the Alexander von Humboldt Foundation. M. Kadler has been supported in part by a Fellowship of the International Max Planck Research School for Radio and Infrared Astronomy and in part by an appointment to the NASA Postdoctoral Program at the Goddard Space Flight Center, administered by Oak Ridge Associated Universities through a contract with NASA. D. Homan is supported by a grant from Research Corporation. The Very Long Baseline Array is operated by the National Radio Astronomy Observatory, a facility of the National Science Foundation operated under cooperative agreement by Associated Universities, Inc. This research has made use of the NASA/IPAC Extragalactic Database (NED), which is operated by the Jet Propulsion Laboratory, California Institute of Technology, under contract with the National Aeronautics and Space Administration.

\section{APPENDIX}

\section{MONTE CARLO CALCULATIONS}

M. Lister et al. (2007, in preparation) perform Monte Carlo calculations to simulate observations being made in the MOJAVE survey (Lister \& Homan 2005). One of their simulations that illustrates the probability densities we need is used here. The calculation selects 100,000 sources with $S>1.5 \mathrm{Jy}(S>2$ Jy in the south) from a large parent population with a power-law distribution of Lorentz factors with index -1.25 and peak $\gamma=32$ and a power-law distribution of intrinsic luminosities with index -2.73 and minimum luminosity $1 \times 10^{24} \mathrm{~W} \mathrm{~Hz}^{-1}$. The model uses an evolving luminosity function based on a fit to the quasars in the CaltechJodrell Bank $6 \mathrm{~cm}$ survey (LM97). The calculation does not assume any correlation between the intrinsic quantities $\gamma$ and $L_{0}$.

Figure 9 shows 14,000 of the selected sources in $(\theta, \gamma)$-space. The curve $\gamma \sin \theta=1.0$ is shown, and it can be seen that the majority of sources have $\theta<\theta_{c}$. The lines $\gamma \sin \theta=0.15$ (lower) and $\gamma \sin \theta=2.0$ (upper) are also shown; these show the 4\% and $96 \%$ cumulative levels for the simulation. They are used in the text as practical limits.

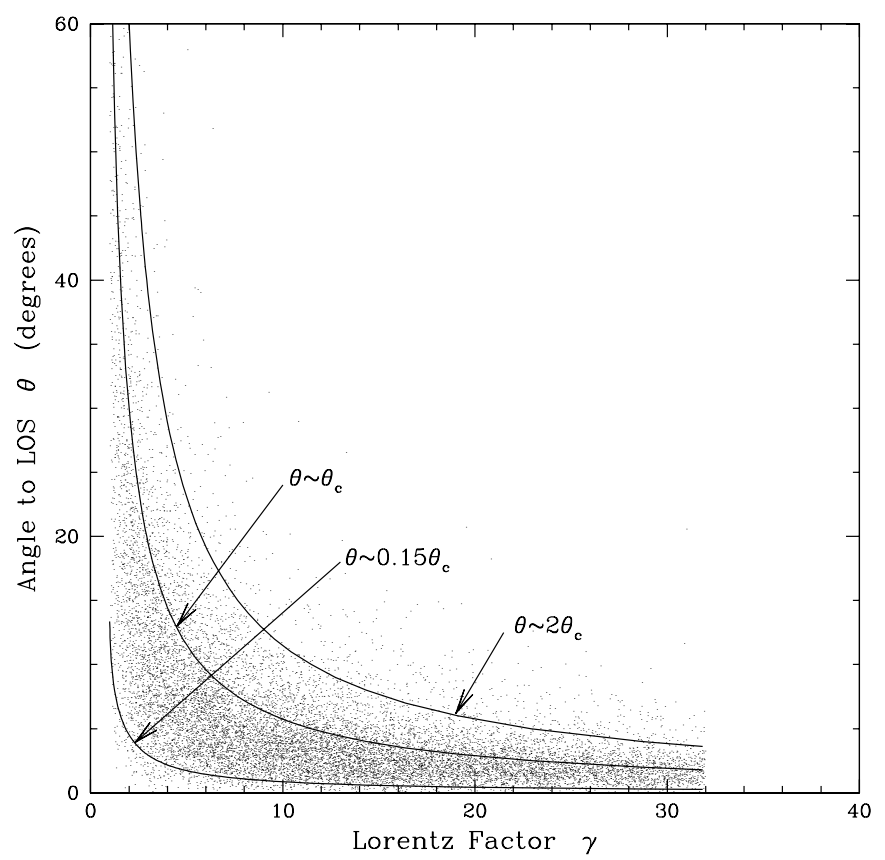

FIg. 9.-Distribution of 14,000 sources selected randomly from the simulation. The central line is $\gamma \sin \theta=1$, or $\theta \approx \theta_{c}$. The upper and lower lines are $\gamma \sin \theta=2.0$ and $\gamma \sin \theta=0.15$, corresponding to $P(\theta)=96 \%$ and $4 \%$, respectively. 
M. Lister et al. (2007, in preparation) discuss the distribution functions for various intrinsic and observed quantities. Here we only show results for subsamples representing slices through the full distribution at constant $\gamma$ and at constant $\beta_{\text {app. }}$. Figure $1 c$ shows a slice for $14.5<\gamma<15.5(N=3638)$; the histograms indicate the probability density, $p(\theta \mid \gamma \approx 15)$, and the cumulative probability, $P(\theta \mid \gamma \approx 15)$. The probability density $p\left(\theta \mid \gamma_{f}\right)$ varies slowly with $\gamma$, and Figure $2 a$ shows $p\left(\sin \theta<\gamma^{-1}\right)$, the expected fraction of sources that will lie within their critical angle. Figures $1 d$ and $2 b$ show similar distributions for $14.5<\beta_{\text {app }}<15.5(N=3191)$.

Agudo, I., Gómez, J.-L., Marti, J.-M., Abanez, J.-M., Marscher, A. P., Alberdi, A., Aloy, M.-A., \& Hardee, P. E. 2001, ApJ, 549, L183

Aller, M. F., Aller, H. D., \& Hughes, P. A. 2003, in ASP Conf. Ser. 300, Radio Astronomy at the Fringe, ed. J. A. Zensus, M. H. Cohen, \& E. Ros (San Francisco: ASP), 159

Antonucci, R. R. J., \& Miller, J. S. 1985, ApJ, 297, 621

Bartel, N., Sorathia, B., Bietenholtz, M. F., Carilli, C. L., \& Diamond, P. 1995, Proc. Natl. Acad. Sci., 92, 11371

Blandford, R. D., \& Königl, A. 1979, ApJ, 232, 34

Chiaberge, M., Celotti, A., Capetti, A., \& Ghisellini, G. 2000, A\&A, 358, 104

Cohen, M. H., Russo, M. A., Homan, D. C., Kellermann, K. I., Lister, M. L., Vermeulen, R. C., Ros, E., \& Zensus, J. A. 2003, in ASP Conf. Ser. 300, Radio Astronomy at the Fringe, ed. J. A. Zensus, M. H. Cohen, \& E. Ros (San Francisco: ASP), 177

Denn, G. R., Mutel, R. L., \& Marscher, A. P. 2000, ApJS, 129, 61

de Vries, W. H., Barthel, P. D., \& O’Dea, C. P. 1997, A\&A, 321, 105

Dondi, L., \& Ghisellini, G. 1995, MNRAS, 273, 583

Giovannini, G., Cotton, W. D., Feretti, L., Lara, L., \& Venturi, T. 2001, ApJ, 552,508

Gómez, J.-L., Marscher, A. P., Alberdi, A., Jorstad, S. G., \& Agudo, I. 2001, ApJ, 561, L161

Homan, D. C., Lister, M. L., Kellermann, K. I., Cohen, M. H., Ros, E., Zensus,

J. A., Kadler, M., \& Vermeulen, R. C. 2003, ApJ, 589, L9

Homan, D. C., Ojha, R., Wardle, J. F. C., Roberts, D. H., Aller, M. F., Aller, H. D., \& Hughes, P. A. 2001, ApJ, 549, 840 2002, ApJ, 568, 99

Homan, D. C., et al. 2006, ApJ, 642, L115

Jorstad, S. G., et al. 2005, AJ, 130, 1418 (J05)

Kellermann, K. I., Vermeulen, R. C., Zensus, J. A., \& Cohen, M. H. 1998, AJ, 115, 1295 (Paper I)

\section{EFERENCES}

Kellermann, K. I., et al. 2004, ApJ, 609, 539 (Paper III)

Kovalev, Y. Y., Nizhelsky, N. A., Kovalev, Y. A., Berlin, A. B., Zhekanis, G. V., Mingaliev, M. G., \& Bogdantsov, A. V. 1999, A\&AS, 139, 545

Kovalev, Y. Y., et al. 2005, AJ, 130, 2473 (Paper IV)

Kraus, A., et al. 2003, A\&A, 401, 161

Lähteenmäki, A., \& Valtaoja, E. 1999, ApJ, 521, 493

Laing, R. A. 1988, Nature, 331, 149

Lind, K. R., \& Blandford, R. D. 1985, ApJ, 295, 358

Lister, M. L., \& Homan, D. C. 2005, AJ, 130, 1389

Lister, M. L., \& Marscher, A. P. 1997, ApJ, 476, 572 (LM97)

Lobanov, A. P. 1998, A\&A, 330, 79

Lovell, J. E. J., Jauncey, D. L., Bignall, H. E., Kedziora-Chudczer, L., Macquart, J.-P., Rickett, B. J., \& Tzioumis, A. K. 2003, AJ, 126, 1699

Ogle, P. M., Cohen, M. H., Miller, J. S., Tran, H. D., \& Goodrich, R. W. 1997, ApJ, 482, L37

Piner, B. G., Mahmud, M., Fey, A. L., \& Gospodinova, K. 2006, AJ, submitted Taylor, G. B., Vermeulen, R. C., Readhead, A. C. S., Pearson, T. J., Henstock, D. R., \& Wilkinson, P. N. 1996, ApJS, 107, 37

Teräsranta, H., Wiren, S., Koivisto, P., Saarinen, V., \& Hovatta, T. 2005, A\&A, 440,409

Urry, C. M., \& Padovani, P. 1995, PASP, 107, 803

Vermeulen, R. C. 1995, Proc. Natl. Acad. Sci., 92, 11385

Vermeulen, R. C., \& Cohen, M. H. 1994, ApJ, 430, 467 (VC94)

Véron-Cetty, M.-P., \& Véron, P. 2003, A\&A, 412, 399

Vestergard, M., \& Barthel, P. D. 1993, AJ, 105, 456

Zensus, A., Ros, E., Kellermann, K. I., Cohen, M. H., Vermeulen, R. C., \& Kadler, M. 2002, AJ, 124, 662 (Paper II) 